\title{
Preditores de prováveis transtornos mentais comuns (TMC) em prostitutas utilizando o Self-Reporting Questionnaire
}

\author{
Predictors of probable common mental disorders (CMD) in \\ sex workers using the Self-Reporting Questionnaire \\ Carlos Eduardo Leal Vidal', Bruna Amara', Daniel Pícoli Ferreira', Isabela Maria Franco Dias', \\ Luana Azevedo Vilela', Luisa Rosa Franco'
}

\section{RESUMO}

Objetivo: Avaliar a prevalência de prováveis transtornos mentais comuns (TMC) e os fatores associados em um grupo de prostitutas de Minas Gerais. Métodos: Estudo transversal utilizando o Self-Reporting Questionnaire (SRQ-20) em mulheres cadastradas na Associação de Prostitutas de Minas Gerais (Aprosmig). Avaliaram-se características sociodemográficas e aspectos do trabalho na prostituição. Teste do qui-quadrado foi empregado na análise de associação entre variáveis categóricas sociodemográficas e a presença de prováveis TMC. A análise dos fatores associados à presença de prováveis TMC foi realizada por meio de modelo de regressão logística. Resultados: Foram entrevistadas 216 prostitutas. A prevalência global de prováveis TMC foi de 57,9\%, mais observada em mulheres com baixa escolarida-

\section{Palavras-chave}

Transtornos mentais, grupos de risco, prostituição.

\section{Keywords}

Mental disorders, risk groups, prostitution. de, história de violência física e ingresso precoce na prostituição. Conclusão: Os resultados deste estudo mostraram que a prevalência de prováveis TMC entre prostitutas foi superior à observada na população geral, indicando a necessidade de melhorar os cuidados com a saúde dessas mulheres.

\begin{abstract}
Objective: To evaluate the prevalence of common mental disorders (CMD) and associated factors in a group of prostitutes in Minas Gerais. Methods: Cross-sectional study using the Self-Reporting Questionnaire (SRQ-20) in women enrolled in the Association of Prostitutes of Minas Gerais (Aprosmig). We assessed sociodemographic characteristics and aspects of work in prostitution. Chi-square test was used to analyze the association between categorical sociodemographic variables and the presence of CMD. Analysis of factors associated with the presence of CMD was performed using logistic regression model. Results: We interviewed 216 prostitutes. The overall prevalence of CMD was $57.9 \%$, more seen in women with low education, history of physical violence and early entry into prostitution. Conclusion: The results of this study showed that the prevalence of CMD among sex workers was higher than that observed in the general population, indicating the need to improve health care of these women.
\end{abstract}

1 Faculdade de Medicina de Barbacena (Fame).

Recebido em

21/3/2014

Aprovado em

$31 / 7 / 2014$ 


\section{INTRODUÇÃO}

Ao longo da vida, a prevalência de transtornos mentais situa-se entre $12 \%$ e 50\%, dependendo da população estudada e dos critérios diagnósticos utilizados ${ }^{1}$. A maioria desses casos, cerca de 90\%, é constituída por transtornos não psicóticos, principalmente transtornos depressivos e transtornos de ansiedade com queixas somáticas, incluindo sintomas como insônia, fadiga, irritabilidade e dificuldade de concentração ${ }^{1,2}$. Esses transtornos, também chamados de transtornos mentais comuns (TMC), são frequentes na população geral e entre pacientes atendidos em serviços de saúde.

A expressão "transtorno mental comum" foi criada por Goldberg e Huxley para caracterizar sintomas como insônia, fadiga, irritabilidade, esquecimento, dificuldade de concentração, queixas somáticas e sintomas depressivos e ansiosos ${ }^{3}$. Esses sintomas trazem incapacidade funcional e sofrimento para seus portadores, embora não preencham os critérios formais para diagnóstico de transtornos do humor (por exemplo, episódios depressivos) e/ou de transtornos específicos de ansiedade4.

Embora os transtornos mentais não sejam causa significativa de mortalidade, são responsáveis por mais de 12\% da incapacitação decorrente de doenças, principalmente em mulheres, tanto em países desenvolvidos como naqueles em desenvolvimento. Além disso, os TMC são associados a piores condições socioeconômicas 5 .

O fato de essas condições não se encaixarem em categorias diagnósticas estabelecidas não diminui sua importância, pois os sintomas provocam sofrimento e aumentam a procura por consultas e exames, muitas vezes desnecessários ${ }^{6}$. Por isso e de acordo com as iniciativas de reorganização da política pública brasileira de saúde mental, alguns autores consideram importante incluir os TMC como uma prioridade na atenção primária à saúde, tal como acontece com outras morbidades crônicas?.

Especificamente com relação ao sexo feminino, parece haver uma predisposição sociobiológica para o surgimento de algumas patologias psiquiátricas, principalmente os TMC. Acredita-se que exista relação entre o sistema neuroendócrino e o papel social da mulher, que interagem de forma a aumentar a vulnerabilidade ${ }^{8}$. As mulheres experimentam taxas mais elevadas de transtornos do humor e transtornos de ansiedade que os homens, e isso está associado a variáveis relativas às condições de vida, às características sociodemográficas e à estrutura ocupacional ${ }^{3,9}$.

As profissionais do sexo, como um grupo marginalizado, parecem apresentar saúde mais debilitada que outros grupos comparáveis da população. Isso se deve a fatores como longas jornadas de trabalho, maior exposição à violência física ou sexual, condições precárias de trabalho e dificuldade de acesso ao sistema de saúde ${ }^{10-13}$. Estudo realizado na Escócia desmascara o mito de que as DST são o principal proble- ma de saúde das trabalhadoras do sexo, acrescentando que os profissionais da saúde precisam repensar a prestação de cuidados para esse grupo ${ }^{14}$.

No Brasil ainda é difícil precisar as necessidades das profissionais do sexo, uma vez que existem poucos estudos englobando os problemas inerentes a esse público, em parte por causa do estigma. Tal fato gera dificuldades na condução de políticas e estratégias de educação em saúde no que diz respeito às concepções de saúde-doença, comportamentos preventivos para infecções e utilização dos serviços de saúde. Ademais, aspectos de vida e saúde das prostitutas são desconhecidos, em decorrência da carência de estudos específicos nessa área ${ }^{15}$.

Considerando que sintomas psiquiátricos são mais prevalentes entre as mulheres e que poucos estudos avaliaram os fatores associados à sua ocorrência em grupos populacionais específicos, o presente estudo tem por objetivo avaliar a prevalência de prováveis TMC entre as profissionais do sexo e descrever os fatores associados à presença dos referidos transtornos.

\section{MÉTODOS}

\section{Tipo de estudo}

Estudo transversal, em que se verificou a prevalência de prováveis TMC em mulheres profissionais do sexo cadastradas na Associação de Prostitutas de Minas Gerais (Aprosmig), utilizando o Self-Reporting Questionnaire (SRQ-20), no período de novembro de 2012 a maio de 2013.

\section{Local do estudo}

O estudo foi realizado na sede da associação, localizada na região central de Belo Horizonte. A Aprosmig é uma entidade sem fins lucrativos, fundada em 2009, que atua nas áreas de promoção da cidadania e busca por direitos das mulheres prostitutas, segurança no trabalho e reconhecimento da profissão. A associação conta com aproximadamente 600 mulheres cadastradas e é referência para as profissionais do sexo do estado de Minas Gerais. A associação possui instalações necessárias para a realização das entrevistas, garantindo-se a privacidade das entrevistadas e o sigilo das informações.

\section{Instrumento de medida}

O SRQ-20 é um questionário destinado à identificação de distúrbios psiquiátricos em nível de atenção primária, desenvolvido por Harding et al. e validado no Brasil por Mari e Willians (apud Gonçalves ${ }^{1}$ e Maragno²) e, posteriormente, por Gonçalves et al., que obtiveram, em seu estudo ${ }^{16}$, sensibilidade de $86,3 \%$ e especificidade de $89,3 \%$ para detecção de TMC, com ponto de corte 7/8, o mesmo empregado no presente trabalho. Utilizado em diversos países, é indicado para 
estudos de base populacional, cujo objetivo seja a identificação de casos psiquiátricos não psicóticos. Possui 20 questões para rastrear sintomas psíquicos e serve de ferramenta para estudos de prevalência2,16,17. Permite discriminar casos positivos de negativos e possui efetividade para o uso em larga escala. Trata-se de instrumento barato, de fácil entendimento (incluindo populações com baixo nível de instrução) e de rápida aplicação. Não necessita de um entrevistador clínico ${ }^{18}$.

Além do SRQ, as participantes responderam a questões relacionadas a variáveis socioeconômicas e clínicas. As variáveis independentes investigadas foram: idade, renda, escolaridade, presença de parceiro fixo, tabagismo, uso de bebidas (frequência semanal e quantidade ingerida), uso de drogas ilícitas, doenças clínicas, filhos, abortos, número de parceiros por semana, conhecimento da profissão por familiares, idade de ingresso na profissão, história de violência física e sexual.

\section{Procedimentos}

O questionário foi aplicado pelos autores, devidamente treinados para a sua aplicação. A amostra foi calculada considerando-se uma prevalência de 30\% de TMC, nível de significância de 5\% e poder de 80\%, totalizando 211 mulheres. Foram entrevistadas as mulheres que se encontravam na referida associação de segunda a sexta-feira, pois nesses dias as prostitutas compareciam ao local para buscar preservativos e outros produtos fornecidos a elas.

\section{Análise estatística}

Foi realizada estatística descritiva para caracterização da amostra segundo variáveis de interesse do estudo e construídas tabelas para distribuição de frequências, médias, medianas e desvio-padrão para cada variável. Testes de qui-quadrado de Pearson assintótico ou exato foram empregados para análise de associação entre variáveis categóricas sociodemográficas e a presença de TMC. A análise dos fatores associados à presença de prováveis TMC foi realizada por meio de modelo de regressão logística. Todas as variáveis que foram significativas com nível de 0,25 foram consideradas candidatas ao modelo multivariado. Inicialmente, foi ajustado um modelo com todas essas variáveis candidatas e, passo a passo, foram retiradas aquelas menos significativas, até ficarem no modelo somente as variáveis significativas com nível de 0,05 . A adequacidade do modelo foi avaliada pelo teste de Hosmer-Lemeshow. Os dados de cada participante foram registrados em fichas elaboradas especialmente para o estudo e posteriormente digitados em planilhas do programa Excel. A análise estatística foi realizada no software SPSS, versão 17.0.

\section{Questões éticas}

Os autores cumpriram os princípios éticos contidos na Declaração de Helsinki. O projeto foi aprovado pelo Comitê de Ética em Pesquisa (CEP) da Universidade Presidente Antônio Carlos (Unipac), Barbacena, MG, em 30/8/2012, Parecer nº
96.923. Todas as participantes assinaram um termo de consentimento livre e esclarecido.

\section{Critérios de inclusão e exclusão}

Os critérios de inclusão para participar do trabalho foram: ser mulher e profissional do sexo cadastrada na Aprosmig, além de assinar um termo de consentimento livre e esclarecido. Travestis, garotos de programa e transexuais, bem como muIheres com idade inferior a 18 anos, não foram incluídos na amostra.

\section{RESULTADOS}

A amostra final foi constituída por 216 mulheres cadastradas na Aprosmig, de um total de 228 abordadas. As principais justificativas apontadas para a não participação de algumas profissionais foram a necessidade de retorno ao trabalho e o medo de serem reconhecidas por familiares por causa do estudo.

O perfil sociodemográfico da amostra estudada aponta mulheres com idade média de $35 \pm 9,9$ anos, com faixa etária variando entre 20 e 68 anos, das quais 68,6\% tinham idade até 40 anos. Evidenciou-se que $65,3 \%$ das prostitutas possuíam ensino médio completo ou nível superior, seguidas por aquelas com tempo de ensino inferior a oito anos, e entre estas $5,1 \%$ nunca frequentaram a escola. A renda per capita média para a maioria $(97,2 \%)$ foi acima de quatro salários-mínimos (considerando o valor de $\mathrm{R} \$ 622,00)$. Na classificação de raças, $34,8 \%$ autorreferiram ter cor da pele parda, seguidos de $33,8 \%$ branca, 29,1\% preta, e somente 2,3\% declararam-se indígenas ou amarelas.

No que diz respeito às variáveis relacionadas ao trabalho, a maioria das profissionais $(49,1 \%)$ relatou uma carga semanal de sete dias trabalhados, atuando em horário matutino, vespertino e/ou noturno, com média de atendimento de 149,5 clientes semanalmente; 93,5\% usavam preservativo masculino nas relações sexuais com os clientes. Mais da metade das prostitutas $(57,4 \%)$ utilizava bebidas alcoólicas durante a jornada de trabalho; em relação ao uso de drogas ilícitas, apenas 18\% referiram a utilização delas para trabalhar. No que se refere ao apoio na realização dessa profissão, 45,4\% afirmaram que a família sabe a respeito da atividade exercida; além disso, 89,8\% das profissionais do sexo manifestaram o desejo de abandonar a prostituição para se dedicarem a outras atividades. Os dados relativos à constituição da amostra estão dispostos na tabela 1 .

Os dados concernentes à prevalência de prováveis TMC nessas mulheres foram correlacionados às variáveis dos perfis sociodemográficos. A prevalência global de prováveis TMC obtida por meio do SRQ foi de 57,9\% (IC 95\%: 51,2\%-64,3\%) entre as prostitutas estudadas. As variáveis que se mostraram associadas à presença de prováveis TMC foram a baixa escolaridade, a história de violência física e o início precoce na prostituição. Esses dados estão apresentados na tabela 2. 
Entre os sintomas listados no Self-Reporting Questionnai$r e$, conforme agrupamento realizado em outro estudo, observou-se que o grupo de sintomas predominantes foi o de "sintomas somáticos" (Tabela 3). Nesse grupo, a maioria das questões obteve elevados percentuais. A questão com maior percentual de respostas afirmativas foi "dorme mal" (58,3\%), seguida de "falta de apetite" (45,3\%). "Humor depressivo/ ansioso" representou o segundo grupo de sintomas mais referidos, com destaque para "sentir-se triste ultimamente" $(50,9 \%)$ e "chorar mais que o costume" (40,2\%). Observou-se que os sintomas relacionados ao desgaste físico e psíquico também foram expressivos. No grupo de "Decréscimo de energia vital", dificuldade em tomar decisões obteve a maior prevalência (43,5\%), enquanto a referência ao pensamento claro apresentou o menor percentual (30,0\%). Sentir-se uma pessoa inútil, sem préstimo $(43,0 \%)$ e ter ideia suicida $(42,1 \%)$ foram os mais citados no grupo "Pensamentos depressivos".

$\mathrm{Na}$ análise multivariada do modelo final de regressão logística, permaneceram as variáveis escolaridade e violência física. Ter menos de oito anos de estudo (OR =2,05; IC 95\%, 1,12-3,73) e história de violência física ( $O R=2,18$; IC 95\%, 1,04-4,56) se associou com maior chance de desenvolvimento de prováveis TMC. O modelo mostrou bom ajuste aos dados conforme o teste de Hosmer-Lemeshow ( $p=0,933)$.

\section{DISCUSSÃO}

$\mathrm{Na}$ amostra estudada, cerca de $60 \%$ das mulheres apresentaram sintomas indicativos de prováveis TMC, percentual bem mais elevado que o encontrado na população geral. No Brasil, a prevalência populacional de TMC observada em diversos estudos variou em torno de 38\%1,3,19, ocorrendo com maior frequência em mulheres (acima de 40\%)2,3,17.

Com relação às prostitutas, não foram localizados estudos avaliando exclusivamente a prevalência de TMC. Um estudo realizado no Rio Grande do Sul (RS) observou prevalência de sintomas depressivos em $67 \%$ das prostitutas avaliadas ${ }^{20}$.

No plano internacional, a prevalência de TMC na população situa-se em torno de 25\% (Organização Mundial da Saúde - OMS), próximo ao observado no Brasil. Estudos realizados especificamente com profissionais do sexo mostraram elevada ocorrência de sintomas psicológicos ${ }^{13}$, transtornos psiquiátricos ${ }^{15}$ e tentativas de suicídio ${ }^{19,21}$ nessas mulheres. Em um estudo australiano ${ }^{22}$ utilizando o General Health Questionnaire (GHQ-28), não foram encontradas diferenças nos escores do questionário entre as prostitutas e o grupo de comparação. Porém, a amostra utilizada no referido estudo foi constituída por apenas 29 mulheres.

A ocorrência de prováveis TMC foi maior nas mulheres com baixa escolaridade, nas que sofreram violência física e naquelas que ingressaram mais cedo na profissão. Com relação à escolaridade, sugere-se que a educação tenha um
Tabela 1. Descrição da amostra de acordo com variáveis sociodemográficas - Belo Horizonte (MG), 2013

\begin{tabular}{|c|c|c|}
\hline Variáveis sociodemográficas & $\mathrm{N}$ & Prevalêncica (\%) \\
\hline \multicolumn{3}{|l|}{ Idade (anos) $(\mathrm{N}=216)$} \\
\hline 20 a 30 & 78 & 36,2 \\
\hline 31 a 40 & 70 & 32,4 \\
\hline 41 a 50 & 51 & 23,6 \\
\hline Acima de 50 & 17 & 7,9 \\
\hline \multicolumn{3}{|l|}{$\operatorname{Cor}(\mathrm{N}=216)$} \\
\hline Preta & 63 & 29,1 \\
\hline Branca & 73 & 33,8 \\
\hline Amarela & 2 & 0,9 \\
\hline Parda & 75 & 34,8 \\
\hline Indígena & 3 & 1,4 \\
\hline \multicolumn{3}{|l|}{ Escolaridade ( $(\mathrm{N}=216)$} \\
\hline Acima de 8 anos & 141 & 65,3 \\
\hline Até 8 anos & 75 & 34,7 \\
\hline \multicolumn{3}{|c|}{ Renda própria mensal ( $N=216)$} \\
\hline Até 4 salários & 6 & 2,8 \\
\hline Acima de 4 salários & 210 & 97,2 \\
\hline \multicolumn{3}{|c|}{ Idade de ingresso na prostituição ( $\mathrm{N}=216$ ) } \\
\hline Antes de 21 anos & 55 & 25,5 \\
\hline Após 21 anos & 161 & 74,6 \\
\hline \multicolumn{3}{|c|}{ Carga horária semanal ( $N=216)$} \\
\hline 1 a 3 dias & 32 & 14,8 \\
\hline 4 a 7 dias & 184 & 85,2 \\
\hline \multicolumn{3}{|c|}{ Número semanal de clientes ( $\mathrm{N}=215)$} \\
\hline Até 150 & 147 & 68,4 \\
\hline Acima de 150 & 68 & 31,6 \\
\hline \multicolumn{3}{|l|}{ Parceiro fixo ( $N=213)$} \\
\hline Não & 126 & 58,4 \\
\hline Sim & 87 & 30,2 \\
\hline \multicolumn{3}{|l|}{ Número de filhos ( $(\mathrm{N}=216)$} \\
\hline Não tem & 34 & 15,7 \\
\hline 1 ou mais & 182 & 84,3 \\
\hline \multicolumn{3}{|c|}{ Realizou algum aborto $(\mathrm{N}=216)$} \\
\hline Não & 124 & 57,6 \\
\hline Sim & 92 & 42,3 \\
\hline \multicolumn{3}{|c|}{ História de violência física na profissão ( $N=216)$} \\
\hline Não & 172 & 79,7 \\
\hline Sim & 44 & 20,4 \\
\hline \multicolumn{3}{|c|}{ História de violência sexual na profissão ( $N=213$ ) } \\
\hline Não & 181 & 83,7 \\
\hline Sim & 32 & 14,8 \\
\hline \multicolumn{3}{|c|}{ Uso de bebida alcoólica durante o trabalho $(\mathrm{N}=216)$} \\
\hline Não & 92 & 42,6 \\
\hline $\operatorname{Sim}$ & 124 & 57,4 \\
\hline \multicolumn{3}{|c|}{ Uso de drogas ilícitas durante 0 trabalho $(\mathrm{N}=214)$} \\
\hline Não & 175 & 81,0 \\
\hline $\operatorname{Sim}$ & 39 & 18,0 \\
\hline \multicolumn{3}{|c|}{ Família sabe da profissão (N=214) } \\
\hline Não & 116 & 53,7 \\
\hline Sim & 98 & 45,4 \\
\hline \multicolumn{3}{|c|}{ Deseja deixar a prostituição $(\mathrm{N}=216)$} \\
\hline Não & 22 & 10,2 \\
\hline Sim & 194 & 89,8 \\
\hline Total & & \\
\hline
\end{tabular}


Tabela 2. Transtornos mentais comuns, odds ratio (OR) e intervalos de confiança de 95\%, segundo variáveis sociodemográficas e clínicas - Belo Horizonte (MG), 2013

\begin{tabular}{|c|c|c|c|c|c|c|}
\hline Variáveis sociodemográficas & TMC+ & $\%$ & TMC- & $\%$ & OR-IR 95\% & Valorp \\
\hline \multicolumn{7}{|l|}{ Idade (anos) $(\mathrm{N}=216)$} \\
\hline 20 a 30 & 47 & 60,3 & 31 & 39,7 & $1,34(0,46-3,87)$ & 0,578 \\
\hline 31 a 40 & 40 & 57,1 & 30 & 42,9 & $1,18(0,40-3,43)$ & 0,754 \\
\hline 41 a 50 & 29 & 56,9 & 22 & 43,1 & $1,17(0,38-3,52)$ & 0,777 \\
\hline Acima de 50 & 9 & 52,9 & 8 & 47,1 & 1 & \\
\hline \multicolumn{7}{|l|}{$\operatorname{Cor}(N=216)$} \\
\hline Branca & 41 & 56,2 & 32 & 43,8 & 1 & \\
\hline Preta & 37 & 58,7 & 26 & 41,3 & $1,11(0,56-2,19)$ & 0,762 \\
\hline Parda & 47 & 58,7 & 33 & 41,3 & $1,11(0,58-2,11)$ & 0,746 \\
\hline \multicolumn{7}{|l|}{ Escolaridade $(\mathrm{N}=216)$} \\
\hline Acima de 8 anos & 73 & 51,8 & 68 & 48,2 & 1 & \\
\hline Até 8 anos & 52 & 69,3 & 23 & 30,7 & $2,10(1,16-3,80)$ & 0,013 \\
\hline \multicolumn{7}{|c|}{ Renda própria mensal ${ }^{*}(\mathrm{~N}=216)$} \\
\hline Até 4 salários & 3 & 50 & 3 & 50 & 1 & \\
\hline Acima de 4 salários & 122 & 58,1 & 88 & 41,9 & $1,38(027-7,03)$ & 0,707 \\
\hline \multicolumn{7}{|c|}{ Idade de ingresso na prostituição ( $(\mathrm{N}=216)$} \\
\hline Após 21 anos & 87 & 54 & 74 & 46 & 1 & \\
\hline Antes de 21 anos & 38 & 69,1 & 17 & 30,9 & $1,90(0,99-3,64)$ & 0,051 \\
\hline \multicolumn{7}{|c|}{ Carga horária semanal ( $\mathrm{N}=216$ ) } \\
\hline 4 a 7 dias & 104 & 56,5 & 80 & 43,5 & 1 & \\
\hline 1 a 3 dias & 21 & 65,6 & 11 & 34,4 & $1,46(0,66-3,22)$ & 0,335 \\
\hline \multicolumn{7}{|c|}{ Número semanal de clientes ( $\mathrm{N}=215$ ) } \\
\hline Acima de 150 & 39 & 57,4 & 29 & 42,7 & 1 & \\
\hline Até 150 & 85 & 57,8 & 62 & 42,2 & $1,02(0,57-1,82)$ & 0,948 \\
\hline \multicolumn{7}{|l|}{ Parceiro fixo $(N=213)$} \\
\hline Não & 71 & 56,3 & 55 & 43,7 & 1 & \\
\hline Sim & 53 & 60,9 & 34 & 39,1 & $1,20(0,69-2,10)$ & 0,506 \\
\hline \multicolumn{7}{|l|}{ Número de filhos ( $\mathrm{N}=216)$} \\
\hline Não tem & 17 & 50 & 17 & 50 & 1 & \\
\hline 1 ou mais & 108 & 59,3 & 74 & 40,7 & $0,68(0,32-1,42)$ & 0,313 \\
\hline \multicolumn{7}{|c|}{ Realizou algum aborto ( $N=216)$} \\
\hline Não & 68 & 54,8 & 56 & 45,2 & 1 & \\
\hline Sim & 57 & 62 & 35 & 38 & $0,74(0,43-1,29)$ & 0,295 \\
\hline \multicolumn{7}{|c|}{ História de violência física na profissão ( $N$ = 216) } \\
\hline Não & 93 & 54,1 & 79 & 45,9 & 1 & \\
\hline Sim & 32 & 72,7 & 12 & 27,3 & $2,26(1,09-4,69)$ & 0,025 \\
\hline \multicolumn{7}{|c|}{ História de violência sexual na profissão ( $N=213$ ) } \\
\hline Não & 104 & 57,5 & 77 & 42,5 & 1 & \\
\hline Sim & 21 & 65,6 & 11 & 34,4 & $1,41(0,64-3,10)$ & 0,387 \\
\hline \multicolumn{7}{|c|}{ Uso de bebida alcoólica durante 0 trabalho $(\mathrm{N}=216)$} \\
\hline $\operatorname{Sim}$ & 71 & 57,3 & 53 & 42,7 & 1 & \\
\hline Não & 54 & 58,7 & 38 & 41,3 & $0,94(0,54-1,62)$ & 0,832 \\
\hline \multicolumn{7}{|c|}{ Uso de drogas ilícitas durante 0 trabalho $(\mathrm{N}=214)$} \\
\hline Não & 102 & 58,3 & 73 & 41,7 & 1 & \\
\hline Sim & 23 & 59 & 16 & 41 & $1,02(0,50-2,08)$ & 0,937 \\
\hline \multicolumn{7}{|c|}{ Família sabe da profissão ( $N=214)$} \\
\hline Não & 65 & 56 & 51 & 44 & 1 & \\
\hline Sim & 59 & 60,2 & 39 & 39,8 & $1,18(0,68-2,04)$ & 0,538 \\
\hline \multicolumn{7}{|c|}{ Deseja deixar a prostituição ( $\mathrm{N}=216)$} \\
\hline Não & 10 & 45,5 & 12 & 54,6 & 1 & \\
\hline Sim & 115 & 59,3 & 79 & 40,7 & $1,74(0,71-4,24)$ & 0,213 \\
\hline
\end{tabular}

*: salário-mínimo 
Tabela 3. Prevalência (\%) dos grupos de sintomas psíquicos avaliados pelo Self-Reporting Questionnaire (SRQ) - Belo Horizonte (MG), 2013

\begin{tabular}{|c|c|c|}
\hline Grupo de sintomas & N & Prevalência (\%) \\
\hline \multicolumn{3}{|l|}{ Humor depressivo/ansioso } \\
\hline Sente-se nervosa, tensa ou preocupada & 81 & 37,5 \\
\hline Assusta-se com facilidade & 78 & 36,1 \\
\hline Tem se sentido triste ultimamente & 110 & 50,9 \\
\hline Tem chorado mais do que de costume & 87 & 40,2 \\
\hline \multicolumn{3}{|l|}{ Sintomas somáticos } \\
\hline Tem dores de cabeça frequentes & 77 & 35,6 \\
\hline Dorme mal & 126 & 58,3 \\
\hline Tem sensações desagradáveis no estômago & 83 & 38,4 \\
\hline Tem má digestão & 82 & 37,9 \\
\hline Tem falta de apetite & 98 & 45,3 \\
\hline Tem tremores nas mãos & 75 & 34,7 \\
\hline \multicolumn{3}{|l|}{ Decréscimo de energia vital } \\
\hline Cansa-se com facilidade & 86 & 39,8 \\
\hline Tem dificuldade em tomar decisões & 94 & 43,5 \\
\hline $\begin{array}{l}\text { Tem dificuldades de realizar com satisfação } \\
\text { suas atividades }\end{array}$ & 92 & 42,5 \\
\hline Seu trabalho é penoso & 71 & 32,8 \\
\hline Sente-se cansada o tempo todo & 88 & 40,7 \\
\hline Tem dificuldade de pensar com clareza & 65 & 30,0 \\
\hline \multicolumn{3}{|l|}{ Pensamentos depressivos } \\
\hline $\begin{array}{l}\text { É incapaz de desempenhar papel útil em sua } \\
\text { vida }\end{array}$ & 59 & 27,3 \\
\hline Tem perdido 0 interesse pelas coisas & 89 & 41,2 \\
\hline Tem tido a ideia de acabar com a vida & 91 & 42,1 \\
\hline Sente-se uma pessoa inútil, sem préstimo & 93 & 43,0 \\
\hline
\end{tabular}

efeito positivo direto na saúde mental, pois aumenta as possibilidades de escolhas no âmbito profissional e pessoal, e interfere na autoestima e na busca de novos conhecimentos. Esse conjunto de fatores pode motivar atitudes e comportamentos mais saudáveis, reduzindo, assim, a probabilidade de ocorrência de prováveis TMC ${ }^{2,23}$. Na população geral também se observa maior frequência de TMC em mulheres com baixa escolaridade ${ }^{3,24}$.

A história de violência física se mostrou associada de forma significativa com a presença de prováveis TMC, o que está de acordo com outros trabalhos ${ }^{25}$. A violência é um grande problema de saúde pública, e parte significativa dos problemas de saúde mental pode ser atribuída à violênciaa ${ }^{25,26}$. No Brasil, a ocorrência de TMC em mulheres é duas vezes mais alta entre vítimas de violência física e psicológica do que em mulheres sem histórico de violência ${ }^{25}$. No entanto, apesar de representar um grupo vulnerável ${ }^{10}$, o percentual de prostitutas entrevistadas que referiu violência física foi menor do que o observado para as mulheres em geral, diferentemente do observado em outros estudos ${ }^{27}$. É provável que tal achado esteja relacionado ao fato de que a maioria das ocorrências de violência contra as mulheres seja perpetrada pelo cônjuge ou pessoas próximas $25,26,28$, o que não foi o caso das prostitutas avaliadas, já que quase dois terços delas não tinham parceiro fixo.

Apesar de a associação não ter sido estatisticamente significativa, a prevalência de prováveis TMC foi maior entre as profissionais entrevistadas que relataram abuso sexual, o que é corroborado por outros estudos ${ }^{12,28}$. Na Europa, $11 \%$ das profissionais do sexo foram vítimas de estupro e $22 \%$ sofreram tentativa de estupro por algum cliente ${ }^{12}$. Na trajetória de vida das prostitutas, observa-se que, quando crianças, geralmente, são abusadas pelos pais ou parentes mais próximos; quando maiores, por seus parceiros; e em exercício da profissão, por seus clientes e empregadores ${ }^{26}$. O cenário da prostituição torna essas mulheres mais vulneráveis ao abuso sexual, o que pode acarretar prejuízos cognitivos, emocionais, comportamentais e sociais.

Em relação à idade de início na profissão, verificou-se que mulheres inseridas na prostituição antes dos 21 anos apresentaram maior chance de desenvolver prováveis TMC. Indícios de violência sexual na infância e desamparo dos pais ou responsáveis são exemplos de situações traumatizantes vivenciadas por algumas mulheres, o que pode facilitar o surgimento de prováveis TMC em médio ou longo prazo. Além disso, grande parte das mulheres com iniciação precoce na prostituição não tem oportunidade de estudo ou qualificação profissional, o que as deixa sem opção ou perspectivas futuras de trabalho.

Algumas variáveis não apresentaram correlação com a prevalência de prováveis TMC em prostitutas, incluindo aqui a média semanal de clientes atendidos e a carga horária semanal de trabalho. Entretanto, outros estudos revelaram que a multiplicidade de parceiros dessas profissionais ${ }^{11}$ e a duração da jornada de trabalho das mulheres em geral contribuem também para a sobrecarga da saúde mental da população feminina. Revelou-se, ainda, que o tempo dedicado às tarefas domésticas, a ausência de apoio para a realização destas e o número elevado de filhos podem repercutir negativamente sobre a saúde das mulheres ${ }^{3}$, o que inclui as profissionais do sexo.

Em relação ao uso de álcool, mais da metade das prostitutas entrevistadas relataram fazer uso de álcool por pelo menos um dia da semana. Em um estudo realizado pelo Instituto Nacional de Políticas Públicas do Álcool e Outras Drogas (Inpad), 39\% das mulheres brasileiras declararam fazer uso de bebidas alcóolicas regularmente ${ }^{29}$. Já no âmbito das drogas ilícitas, 18,1\% afirmaram utilizar. A maconha e a cocaína demonstraram ser as drogas ilícitas de maior consumo entre a amostra estudada. No Brasil, a pesquisa realizada pelo Inpad demonstrou que 3\% da população usou maconha no último ano e $2 \%$ dela fez o uso de cocaína e seus derivados ${ }^{30,31}$. 
Este estudo apresenta algumas limitações que devem ser consideradas. Em relação à amostra, o público escolhido foi constituído por profissionais cadastradas na Aprosmig, o que não representa a totalidade das prostitutas mineiras. Essa limitação impede a generalização dos resultados. Além disso, o questionário foi conduzido por diferentes entrevistadores/ pesquisadores, que, apesar de devidamente treinados para sua aplicação, possuem características individuais na condução da entrevista. Com relação ao uso de bebidas alcoólicas, não foi utilizado nenhum instrumento validado para mensurar a quantidade ingerida e a frequência de uso.

Outros fatores que podem ter sido limitantes foram as características momentâneas das entrevistadas (estado de fadiga, saúde, distração) e possíveis omissões ou respostas inverídicas por medo de julgamento, vitimização e pressa para retornar às suas atividades. A maximização dos sintomas relatados pelas participantes pode estar presente e constitui um importante viés na coleta de dados da pesquisa, já que algumas prostitutas complementaram as respostas objetivas correlacionando o sofrimento apresentado à estigmatização a que são submetidas. Esse processo talvez seja evidenciado com maior nitidez no estudo qualitativo proposto pelos pesquisadores, por meio da avaliação do discurso dessas mulheres, conforme observado por outros autores $^{32}$.

E, por fim, o fato de as entrevistas terem sido realizadas apenas com as mulheres que se encontravam na referida associação pode se configurar como um viés de seleção, o que poderia alterar os resultados encontrados em uma ou outra direção. Aquelas que estavam no local e aceitaram participar da pesquisa poderiam constituir um grupo de mulheres com menor número de sintomas e problemas relacionados ao trabalho, diminuindo a prevalência. Ou, ao contrário, ao saber dos objetivos da pesquisa, algumas mulheres portadoras de sintomas físicos e/ou psíquicos poderiam ter se apresentado como voluntárias e elevado os coeficientes observados ${ }^{33}$.

\section{CONCLUSÃO}

Os resultados deste estudo mostraram que a prevalência de prováveis TMC em prostitutas foi superior à observada na população geral. A elevada ocorrência de prováveis TMC nesse grupo de mulheres aponta para a necessidade de meIhorar os cuidados com a sua saúde, tanto nos aspectos preventivos quanto de tratamento.

O ingresso precoce na prostituição e a baixa escolaridade das mulheres merecem ser destacados. As duas variáveis se mostraram associadas à ocorrência de prováveis TMC e, provavelmente, interagindo de forma recíproca no seu surgimento. Tanto o nível de escolaridade baixo quanto o ingresso cedo na prostituição são condições que limitam a qualificação profissional e as perspectivas futuras de trabalho.
Outro dado importante diz respeito ao componente da violência, o que não é atributo exclusivo das prostitutas, mas de boa parcela da população feminina. Apesar de a legislação proteger as mulheres e penalizar os agressores, o número de vítimas de atos violentos ainda é expressivo.

No presente estudo, as entrevistadas não foram questionadas sobre o acesso e o acolhimento nos serviços de saúde e nem como lidam com situações de violência, estigma e discriminação. Esses tópicos serão abordados em estudo futuro, de ordem qualitativa, procurando aprofundar a análise por meio da interpretação de narrativas de algumas prostitutas selecionadas da amostra inicial.

\section{CONTRIBUIÇÕES INDIVIDUAIS}

Carlos Eduardo Leal Vidal - Contribuiu na concepção, no desenho, na análise e na interpretação dos dados, na elaboração do artigo e na aprovação da versão final.

Todos os demais autores contribuíram significativamente na concepção, no desenho, na coleta e na interpretação dos dados, na elaboração do artigo e na aprovação da versão final a ser publicada.

\section{CONFLITOS DE INTERESSE}

Os autores declaram não haver conflitos de interesse.

\section{REFERÊNCIAS}

1. Gonçalves DM, Kapczinski F. Transtornos mentais em comunidade atendida pelo Programa Saúde da Família. Cad Saude Publica. 2008;24(7):1641-50.

2. Maragno L, Goldbaum M, Gianini RJ, Novaes MDH, César LGC. Prevalência de transtornos mentais comuns em populações atendidas pelo Programa Saúde da Família (QUALIS) no município de São Paulo, Brasil. Cad Saude Publica. 2006;22(8):1639-48.

3. Araújo TM, Pinho PS, Almeida MMG. Prevalência de transtornos mentais comuns em muIheres e sua relação com as características sociodemográficas e 0 trabalho doméstico. Rev Bras Saúde Matern Infant. 2005;5(3):337-48

4. Baker L, Case P, Policicchio D. General health problems of inner-city sex workers: a pilot study. J Med Libr Assoc. 2003;91(1):67-71.

5. Aquino PS, Nicolau AIO, Pinheiro AKB. Desempenho das atividades de vida de prostitutas segundo o Modelo de Enfermagem de Roper, Logan e Tierney. Rev Bras Enferm. 2011;64(1):136-44.

6. Gomes VF, Miguel TLB, Miasso Al. Common mental disorders: socio-demographic and pharmacotherapy profile. RevLatino-Am Enfermagem. 2013;21(6):1203-11. Disponível em: http://www. scielo.br/scielo.php?script=sci_arttext\&pid=\$0104-11692013000601203\&lng=en.\%20 http://dx.doi.org/10.1590/0104-1169.2990.2355. Acesso em: 18 maio 2014.

7. Gonçalves DA, Mari JJ, Bower P, Gask L, Dowrick C, Tófoli LF, et al. Brazilian multicentre study of common mental disorders in primary care: rates and related social and demographic factors. Cad Saude Publica. 2014;30(3):623-32. Disponível em: http://www.scielosp.org/ scielo.php?script=sci_arttext\&pid=S0102-311X2014000300623\&lng=en.\%20http:// dx.doi.org/10.1590/0102-311X00158412. Acesso em: 18 maio 2014.

8. Andrade LHSG, Viana MC, Silveira CM. Epidemiologia dos transtornos psiquiátricos na muIher. Rev Psiq Clín. 2006;33:43-54. 
9. Martin D, Quirino J, Mari J. Depressão entre mulheres da periferia de São Paulo. Rev Saúde Pública. 2007;41(4):591-7.

10. Rössler W, Koch U, Lauber C, Hass A, Altwegg M, Landolt K, et al. The mental health of female sex workers. Acta Psychiatr Scand. 2010;122(2):143-52.

11. Moraes MLC, Costa PB, Aquino OS, Pinheiro AKB. Educação em saúde com prostitutas de Fortaleza: relato de experiência. Rev Eletr Enf [Internet]. 2008;10(4):1144-51. Disponível em: http://www.fen.ufg.br/revista/v10/n4/v10n4a27.htm.

12. Moreira ICCC, Monteiro CFS. A violência no cotidiano da prostituição: invisibilidades e ambiguidades. Rev Latino-Am Enfermagem. 2012;20(5):954-60.

13. El-Bassel N, Schilling R, Irwin K, Faruque S, Gilbert L, Edlin B, et al. Sex trading and psychological distress among women recruited from the streets of Harlem. Am J Public Health. 1997;87(1):66-70.

14. Carr SV. The health of women working in the sex industry - A moral and ethical perspective. Sex Marital Ther. 1995;10(2):201-13.

15. Brody S, Potterat J. Assessing mental health and personality disorder in prostitute women. Acta Psychiatr Scand. 2010;122(2):167-68.

16. Gonçalves DM, Stein AT, Kapczinski F. Avaliação de desempenho do Self-Reporting Questionnaire como instrumento de rastreamento psiquiátrico: um estudo comparativo com o Structured Clinical Interview for DSM-IV-TR. Cad Saude Publica. 2008;24(2):380-90.

17. lacoponi E, Mari JJ. Reliability and factor structure of the Portuguese version of Self-Reporting Questionnaire. Int J Soc Psychiatry. 1998;35(3):213-22.

18. Potter K, Martin J, Romans S. Early developmental experiences of female sex workers: a comparative study. Aust N Z J Psychiatry. 1999;33(6):935-40.

19. Bandeira M, Freitas LC, Carvalho Filho JGT. Avaliação da ocorrência de transtornos mentais comuns em usuários do Programa de Saúde da Família. J Bras Psiquiatr. 2007;56(1):41-7.

20. Schreiner L, Paim LL, Ramos F, Cunha FEV, Martins DM, Silva CJL, et al. Prevalência de sintomas depressivos em uma amostra de prostitutas de Porto Alegre. Rev Psiquiatr Rio Gd Sul. 2004;26(1):13-20.

21. Shahmanesh M, Wayal S, Cowan F, Mabey D, Copas A, Patel V. Suicidal behavior among female sex workers in Goa, India: the silent epidemic. Am J Public Health. 2009;99(7):1239-46.
22. Romans S, Potter K, Martin J, Herbison P. The mental and physical health of female sex workers: a comparative study. Aust N Z J Psychiatry. 2001;35(1):75-80.

23. Ludermir AB, Melo Filho DA. Condições de vida e estrutura ocupacional associadas a transtornos mentais comuns. Rev Saúde Pública. 2002;36(2):213-21.

24. Rocha SV. Prevalência de transtornos mentais comuns entre residentes em áreas urbanas de Feira de Santana, Bahia. Rev Bras Epidemiol. 2010;13(4):630-40.

25. Ribeiro, WS. Exposição à violência e problemas de saúde mental em países em desenvolvimento: uma revisão da literatura. Rev Bras Psiquiatr. 2009;31(Supl 2):S49-57.

26. Penha JC. Caracterização da violência física sofrida por prostitutas do interior piauiense. Rev Bras Enferm. 2012;65(6):984-90.

27. De Meis C. House and street: narratives of identity in a liminal space among prostitutes in Brazil. Ethos. 2002;30(1-2):3-24.

28. Schraiber LB. Prevalência da violência contra a mulher por parceiro íntimo em regiões do Brasil. Rev Saúde Pública. 2007;41(5):797-807.

29. Laranjeira R, Madruga CS, Pinsky I, Caetano R, Ribeiro M, Mitsuhiro S. II Levantamento Nacional de Álcool e Drogas - Consumo de Álcool no Brasil: Tendências entre 2006/2012. São Paulo: Inpad; 2013 [acesso em 15 setembro 2013]. Disponível em: http://inpad.org.br/ wp-content/uploads/2013/04/LENAD_ALCO0L_Resultados-Preliminares.pdf.

30. Laranjeira R, Madruga CS, Pinsky I, Caetano R, Ribeiro M, Mitsuhiro S. II Levantamento Nacional de Álcool e Drogas - 0 Uso de Maconha no Brasil. São Paulo: Inpad; 2013 [acesso em 15 setembro 2013]. Disponível em: http://inpad.org.br/wp-content/uploads/2013/04/ Press_Maconha_Slte1.pdf.

31. Laranjeira R, Madruga CS, Pinsky I, Caetano R, Ribeiro M, Mitsuhiro S. II Levantamento Nacional de Álcool e Drogas - 0 Uso de Cocaína e Crack no Brasil. São Paulo: Inpad; 2013 [acesso em 15 setembro 2013]. Disponível em: http://inpad.org.br/wp-content/uploads/2013/03/LENAD_PressRelease_Coca.pdf.

32. Garotas de programa: prostituição em Copacabana e identidade social. 2a ed. Rio de Janeiro: Jorge Zahar Editor; 1988.

33. Gordis L. Epidemiologia. Rio de Janeiro: Revinter; 2010. 